\title{
An exact upper bound for sums of element orders in non-cyclic finite groups
}

\author{
Marcel Herzog ${ }^{\mathrm{a}}$, Patrizia Longobardi ${ }^{\mathrm{b}, *}$, Mercede Maj ${ }^{\mathrm{b}}$ \\ ${ }^{a}$ Department of Mathematics, Raymond and Beverly Sackler Faculty of Exact Sciences, \\ Tel Aviv University, Tel Aviv, Israel \\ ${ }^{b}$ Dipartimento di Matematica, Università di Salerno, via Giovanni Paolo II, 132, 84084 \\ Fisciano (Salerno), Italy
}

\begin{abstract}
Denote the sum of element orders in a finite group $G$ by $\psi(G)$ and let $C_{n}$ denote the cyclic group of order $n$. Suppose that $G$ is a non-cyclic finite group of order $n$ and $q$ is the least prime divisor of $n$. We proved that $\psi(G) \leq \frac{7}{11} \psi\left(C_{n}\right)$ and $\psi(G)<\frac{1}{q-1} \psi\left(C_{n}\right)$. The first result is best possible, since for each $n=4 k$, $k$ odd, there exists a group $G$ of order $n$ satisfying $\psi(G)=\frac{7}{11} \psi\left(C_{n}\right)$ and the second result implies that if $G$ is of odd order, then $\psi(G)<\frac{1}{2} \psi\left(C_{n}\right)$. Our results improve the inequality $\psi(G)<\psi\left(C_{n}\right)$ obtained by H. Amiri, S.M. Jafarian Amiri and I.M. Isaacs in 2009, as well as other results obtained by S.M. Jafarian Amiri and M. Amiri in 2014 and by R. Shen, G. Chen and C. Wu in 2015. Furthermore, we obtained some $\psi(G)$-based sufficient conditions for the solvability of $G$.
\end{abstract}

Keywords: Group element orders, Solvable groups

2010 MSC: 20D60, 20E34, 20F16

\section{Introduction}

The problem of detecting structural properties of a periodic group by looking at element orders has been considered by various authors, from many different points of view. For example, if we denote by $\omega(G)$ the set of the orders of all

\footnotetext{
*corresponding author

Email addresses: herzogm@post.tau.ac.il (Marcel Herzog), plongobardi@unisa.it (Patrizia Longobardi), mmaj@unisa.it (Mercede Maj)
} 
5 the elements of $G$, there are many new and old results as well as many open questions concerning $\omega(G)$ (see for example [9]). In [1] H. Amiri, S.M. Jafarian Amiri and I.M. Isaacs introduced the function $\psi(G)$, which denotes the sum of element orders of a finite group $G$, and proved that if $G$ is a non-cyclic group of order $n$ then $\psi(G)<\psi\left(C_{n}\right)$, where $C_{n}$ denote the cyclic group of order $n$. 10 Recently S.M. Jafarian Amiri and M. Amiri in [5] (see also [2] and [4]) and R. Shen, G. Chen and C. Wu in [11] studied finite groups $G$ of order $n$ with the second largest value of $\psi(G)$, and obtained information about the structure of $G$ if $n=p_{1}^{\alpha_{1}} \cdots p_{t}^{\alpha_{t}}, p_{1}<\cdots<p_{t}$, in the case $\alpha_{1}>1$. Products of element orders of a finite group $G$ and some other functions on the orders of the elements of $G$ have been recently studied by M. Garonzi and M. Patassini in [3].

In this paper we continue the study of the function $\psi(G)$.

Our main result is the following theorem:

Theorem 1 If $G$ is a non-cyclic finite group of order $n$, then

$$
\psi(G) \leq \frac{7}{11} \psi\left(C_{n}\right)
$$

This upper bound is best possible, since as shown in the following proposition, for each $n=4 k, k$ odd, there exists a group of order $n$ satisfying $\psi(G)=$ $\frac{7}{11} \psi\left(C_{n}\right)$.

Proposition 2 Let $k$ be an odd integer and let $n=4 k$. Then

$$
\psi\left(C_{n}\right)=11 \psi\left(C_{k}\right) \quad \psi\left(C_{2 k} \times C_{2}\right)=7 \psi\left(C_{k}\right)
$$

and hence

$$
\psi\left(C_{2 k} \times C_{2}\right)=\frac{7}{11} \psi\left(C_{n}\right)
$$

In particular, in view of Theorem 1, it follows by Proposition 2 that if $n=4 k$ with an odd $k$, then the group $G=C_{2 k} \times C_{2}$ has the maximal sum of element 25 orders among non-cyclic groups of order $n$ (see also [11], Theorem 1.1). 
We also proved the following result, which improves Theorem 1 for groups of odd order.

Theorem 3 Let $G$ be a non-cyclic finite group of order $n$ and let $q$ be the smallest prime divisor of $n$. Then:

$$
\psi(G)<\frac{1}{q-1} \psi\left(C_{n}\right) .
$$

Indeed, this theorem implies the following corollary:

Corollary 4 Let $G$ be a non-cyclic finite group of odd order $n$. Then

$$
\psi(G)<\frac{1}{2} \psi\left(C_{n}\right) .
$$

An important ingredient in our proofs is Corollary B of [1], which states that if $P$ is a cyclic normal Sylow $p$-subgroup of a finite group $G$, then

$$
\psi(G) \leq \psi(P) \psi(G / P),
$$

with equality if and only if $P$ is central in $G$ (see Proposition 2.10). Another important ingredient is our Lemma 2.1, where we proved that if $p$ and $q$ are the largest and the smallest divisors of an integer $n$, respectively, then the Euler's function $\varphi(n)$ satisfies the following inequality:

$$
\varphi(n) \geq \frac{q-1}{p} n .
$$

We also mention the following almost trivial upper bound for the value of $\psi(G)$ for non-cyclic groups $G$.

Proposition 5 Let $G$ be a non-cyclic finite group of order $n$ and let $q$ be the smallest prime divisor of $n$. Then:

$$
\psi(G) \leq \frac{(n-1) n}{q}+1<\frac{n^{2}}{q} .
$$


Proof. Since $G$ is non-cyclic, it follows that $o(x) \leq n / q$ for each $x \in G$. But $o(1)=1$, so $\psi(G) \leq(n-1)(n / q)+1<n^{2} / q$, as required.

Notice, however that $\psi\left(S_{3}\right)=13>\frac{1}{2}(q-1) \psi\left(C_{6}\right)=\frac{21}{2}$. This observation raises the following question: what can we say about groups of order $n$ satisfying $\psi(G) \geq(1 / 2(q-1)) \psi\left(C_{n}\right)$ ? A partial answer can be found in our next theorem.

Theorem 6 Let $G$ be a finite group of order $n$ and let $q$ and $p$ be the smallest and the largest prime divisors of $n$, respectively. Suppose that $G$ satisfies

$$
\psi(G) \geq \frac{1}{2(q-1)} \psi\left(C_{n}\right) .
$$

Then $G$ is solvable, the Sylow p-subgroups of $G$ contain a cyclic subgroup of index $p$ and one of the following statements holds:

(i) The Sylow p-subgroup $P$ of $G$ is cyclic and normal in $G$;

(ii) The Sylow q-subgroups of $G$ are cyclic, $G$ is $q$-nilpotent and $G^{\prime \prime} \leq Z(G)$;

(iii) The Sylow p-subgroups of $G$ are cyclic, $G$ is $p$-nilpotent and $G^{\prime \prime} \leq Z(G)$.

Theorem 6 implies the following corollaries. We use $p$ and $q$ as defined in Theorem 6 .

Corollary 7 The conclusions of Theorem 6 hold if $G$ satisfies

$$
\psi(G) \geq \frac{1}{q} \psi\left(C_{n}\right) .
$$

${ }_{45}$ Proof. Since $q \geq 2$, it follows that $q \leq 2(q-1)$.

Corollary 8 The conclusions of Theorem 6 hold if $G$ is a group of odd order satisfying

$$
\psi(G) \geq \frac{1}{q+1} \psi\left(C_{n}\right) .
$$

Proof. Since $q \geq 3$, it follows that $q+1 \leq 2(q-1)$.

Corollary 9 If either $G$ is non-solvable or a Sylow p-subgroup of $G$ contains no cyclic subgroup of index $p$, then

$$
\psi(G)<\frac{1}{2(q-1)} \psi\left(C_{n}\right) \leq \frac{1}{q} \psi\left(C_{n}\right) .
$$


Our next result is another $\psi(G)$-based sufficient condition for the solvability of $G$.

Theorem 10 Let $G$ be a finite group of order $n$ satisfying

$$
\psi(G) \geq \frac{3}{5} n \varphi(n)
$$

Then $G$ is solvable and $G^{\prime \prime} \leq Z(G)$.

This condition is certainly not necessary for the solvability of $G$. For example, for $n=8$ we have

$$
\psi\left(C_{2} \times C_{2} \times C_{2}\right)=15<\frac{3}{5} \cdot 8 \cdot 4=\frac{3}{5} n \varphi(n) .
$$

On the other hand, for $n=60$, the simple group $A_{5}$ satisfies $\psi\left(A_{5}\right)=211>$ $\frac{1}{5} n \phi(n)=192$.

In the proof of Theorem 10 we apply the following result of Ramanujan (see [8], page 46): if $q_{1}=2, q_{2}, \cdots, q_{n}, \cdots$ is the increasing sequence of all primes, then

$$
\prod_{i=1, \cdots, \infty} \frac{q_{i}^{2}+1}{q_{i}^{2}-1}=\frac{5}{2}
$$

Our final result deals with groups of order $n$ which satisfy $\psi(G) \geq \frac{1}{q} n \varphi(n)$.

Theorem 11 Let $G$ be a finite group of order $n$ and let $q$ and $p$ be the smallest and the largest prime divisors of $n$, respectively. Suppose that $G$ satisfies

$$
\psi(G) \geq \frac{1}{q} n \varphi(n) .
$$

Then either $G$ has a normal cyclic Sylow p-subgroup or it is a solvable group ${ }_{55}$ with a cyclic maximal subgroup of index either $p$ or $p+1$.

\section{Acknowledgment}

We are grateful to our colleague Guy Moshkowich for constructing the table of the values of the function $\psi(G)$ for all groups $G$ of order less than 128. This 
table, which he constructed using the GAP System of Computational Group Theory, provided useful information for our research.

This work was supported by the "National Group for Algebraic and Geometric Structures, and their Applications" (GNSAGA - INDAM), Italy.

The first author is grateful to the Department of Mathematics of the University of Salerno for its hospitality and support, while this investigation was carried out.

\section{Preliminary results}

First we determine a lower bound for $\varphi(n)$.

Lemma 2.1 Let $n$ be a positive integer larger than 1, with the largest prime divisor $p$ and the smallest prime divisor $q$. Then

$$
\varphi(n) \geq \frac{q-1}{p} n .
$$

Proof. Let $n=p_{1}^{r_{1}} p_{2}^{r_{2}} \cdots p_{k}^{r_{k}}$, where the $p_{i}$ 's are primes, the $r_{i}$ 's are positive integers and $p=p_{1}>p_{2}>\cdots>p_{k}=q$. Our proof is by induction on $k$.

If $k=1$, then $n=p^{r_{1}}$ and

$$
\varphi(n)=\varphi\left(p^{r_{1}}\right)=\frac{p-1}{p} p^{r_{1}}=\frac{p-1}{p} n,
$$

as required.

Suppose now that $k>1$ and that the lemma holds for all integers with less than $k$ distinct prime divisors. Set $m=p_{2}^{r_{2}} \cdots p_{k}^{r_{k}}$. Then by induction $\varphi(m) \geq \frac{p_{k}-1}{p_{2}} m$ and

$\varphi(n)=\varphi\left(p_{1}^{r_{1}}\right) \varphi(m) \geq \frac{p_{1}-1}{p_{1}} p_{1}^{r_{1}} \frac{p_{k}-1}{p_{2}} m \geq \frac{p_{1}-1}{p_{1}} \frac{p_{k}-1}{p_{1}-1} n=\frac{p_{k}-1}{p_{1}} n=\frac{q-1}{p} n$, as required. The proof is now complete.

Our next aim is to find a convenient formula for $\psi(G)$ when $G=P \rtimes F, P$ is a cyclic $p$-group for some prime $p,|F|>1$ and $(p,|F|)=1$. 
Lemma 2.2 Let $G$ be a finite group satisfying $G=P \rtimes F$, where $P$ is a cyclic p-group for some prime $p,|F|>1$ and $(p,|F|)=1$. Then the following statements hold.

(1) Each element of $F$ acts on $P$ either trivially or fixed-point-freely.

(2) If $x \in F, o(x)=m$ and $u \in P$, then $m$ is the least positive integer satisfying $(u x)^{m} \in P$.

(3) If $u \in P$ and $x \in C_{F}(P)$, then $o(u x)=o(u) o(x)$.

(4) If $u \in P$ and $x \in F \backslash C_{F}(P)$, then $o(u x)=o(x)$.

(5) Let $Z=C_{F}(P)$. Then

$$
\psi(G)=\psi(P) \psi(Z)+|P| \psi(F \backslash Z)<\psi(P) \psi(Z)+|P| \psi(F) .
$$

Proof.

(1) Suppose that $x \in F$ acts trivially on $u \in P \backslash\{1\}$. Then $x$ acts trivially on $\Omega_{1}(P)$ and hence it acts trivially on $P$ (see [6], Theorem 5.4.2). The claim follows from this remark.

(2) Since $P \triangleleft G$, it follows that if $n$ is a positive integer, then $(u x)^{n}=v_{n} x^{n}$ for some $v_{n} \in P$. As $P \cap F=\{1\}$, it follows that $(u x)^{n} \in P$ if and only if $m$ divides $n$. The claim follows.

(3) Trivially holds.

(4) Suppose that $o(x)=m$. By (2) $(u x)^{m} \in P$ and hence $1=\left[(u x)^{m}, u x\right]=$ $\left[(u x)^{m}, x\right]$. Since $x \in F \backslash C_{F}(P)$, it follows by (1) that $(u x)^{m}=1$ and by (2) $o(u x)=m=o(x)$, as required.

(5) It follows by (3) that $\psi(P Z)=\psi(P) \psi(Z)$ and by (4) that $\psi(G \backslash(P Z))=$ $|P| \psi(F \backslash Z)$. Therefore

$$
\psi(G)=\psi(P) \psi(Z)+|P| \psi(F \backslash Z)<\psi(P) \psi(Z)+|P| \psi(F) .
$$

We also need information concerning finite groups with a cyclic maximal subgroup. First we mention the following related result of Herstein [7]. 
Proposition 2.3 (Herstein) If $G$ is a finite group with an abelian maximal subgroup, then $G$ is solvable.

Using this result we proved the following proposition, which is of independent interest.

Proposition 2.4 Let $G$ be a finite group with a cyclic maximal subgroup $C$. Then $G$ is solvable and $G^{\prime \prime} \leq Z(G)$.

Proof. The group $G$ is solvable by Proposition 2.3. If $G^{\prime} \leq C$, then $G^{\prime \prime}=1 \leq$ $Z(G)$ as required. Otherwise $G=G^{\prime} C$ and $G^{\prime \prime} \leq C$, since otherwise $G=G^{\prime \prime} C$ and $G^{\prime} \leq G^{\prime \prime}$, a contradiction since $G$ is solvable and $G^{\prime} \neq 1$. Hence $G^{\prime \prime}$ is cyclic and $G / C_{G}\left(G^{\prime \prime}\right)$ is abelian. Consequently $G^{\prime}$ and $C$ are both subgroups of $C_{G}\left(G^{\prime \prime}\right)$, yielding again $G^{\prime \prime} \leq Z(G)$, as required.

Another important and useful result is the following proposition.

Proposition 2.5 Let $G$ be a finite group and suppose that there exists $x \in G$ such that

$$
[G:\langle x\rangle]<2 p,
$$
where $p$ is the maximal prime divisor of $|G|$. Then one of the following holds:

(i) G has a normal cyclic Sylow p-subgroup,

(ii) $G$ is solvable and $\langle x\rangle$ is a maximal subgroup of $G$ of index either $p$ or $p+1$.

Proof. First suppose that $p$ divides $[G:\langle x\rangle]$. Since $[G:\langle x\rangle]$ divides $|G|$, our assumption implies that $[G:\langle x\rangle]=p$ and $G$ is solvable by Proposition 2.4. Thus $G$ satisfies (ii).

Now assume that $p$ does not divide $[G:\langle x\rangle]$. Then $\langle x\rangle$ contains a cyclic Sylow $p$-subgroup $P$ of $G$. If $P$ is normal in $G$, then (i) holds. So suppose, finally, that $P$ is not normal in $G$. Since $\langle x\rangle \leq N_{G}(P)$, it follows from our 
assumptions that $\left[G: N_{G}(P)\right]<2 p$. Since $P$ is not normal in $G$, this implies that $\left[G: N_{G}(P)\right]=p+1$ and that $N_{G}(P)$ is a maximal subgroup of $G$. But

$$
\left[N_{G}(P):\langle x\rangle\right]=\frac{[G:\langle x\rangle]}{\left[G: N_{G}(P)\right]}<\frac{2 p}{p+1}<2,
$$

so $N_{G}(P)=\langle x\rangle$ and $\langle x\rangle$ is a cyclic maximal subgroup of $G$ of index $p+1$. By Proposition $2.4 G$ is solvable, and hence it satisfies (ii). The proof of the proposition is complete.

We also need the following related result.

Proposition 2.6 The following statements hold.

(1) If $G$ is a finite 2-group with a cyclic subgroup of index 4 , then $G^{\prime \prime} \leq Z(G)$.

(2) If $G$ is a finite group of order $2^{\alpha} 3^{\beta}$ with a cyclic subgroup of index less than 6 , then $G^{\prime \prime} \leq Z(G)$.

Proof. (1) Let $\langle a\rangle$ be of index 4 in $G$ and let $M$ be a maximal subgroup of $G$ containing $\langle a\rangle$. If $M=\langle a\rangle$, then the result follows by Proposition 2.4. So assume that $M>\langle a\rangle$. Then $[G: M]=2$, implying that $M$ is normal in $G, G^{\prime} \leq M$ and $G^{\prime \prime} \leq M^{\prime}$. Moreover, $M$ has a maximal cyclic subgroup and therefore by Theorem 5.3.4 in [10], either $M$ is abelian, or $M^{\prime}$ has order 2, or $M$ is dihedral, semidihedral or generalized quaternion.

If $M$ is abelian then $G^{\prime \prime}=1$ and if $\left|M^{\prime}\right| \leq 2$, then $G^{\prime \prime} \leq M^{\prime}$ implies that $G^{\prime \prime}$ is a normal subgroup of $G$ of order at most 2 , thus $G^{\prime \prime} \leq Z(G)$, as required.

Now suppose that $M$ is either dihedral, or semidihedral or generalized quaternion. Then there exists $x \in M$ such that $a^{x}=a^{-1} a^{\gamma 2^{n-1}}$, where $o(a)=2^{n}$, ${ }_{135} \gamma \in\{0,1\}, o(x) \in\{2,4\}$ and $x^{2} \in Z(M)$.

Write $G=M\langle y\rangle$.

If $a^{y} \in\langle a\rangle$, then $\langle a\rangle$ is normal in $G$, thus $G^{\prime} \leq\langle a\rangle$ since $|G /\langle a\rangle|=4$. Hence $G^{\prime}$ is abelian and $G^{\prime \prime}=1$, as required.

Suppose, finally, that $a^{y} \notin\langle a\rangle$. Then $a^{y}=a^{\delta} x$, where $\delta$ is an integer. We have

$$
\left(a^{2}\right)^{y}=a^{\delta} x a^{\delta} x=a^{\delta} x^{2} a^{-\delta} a^{\gamma 2^{n-1} \delta}=x^{2} a^{\gamma 2^{n-1} \delta}
$$


and $\left(a^{y}\right)^{4}=\left(a^{\gamma 2^{n-1}} \delta x^{2}\right)^{2}=x^{4}=1$. Hence $o(a)=o\left(a^{y}\right)=4,|M|=8$ and $M^{\prime} \leq\left\langle a^{2}\right\rangle$. Thus $G^{\prime \prime} \leq M^{\prime}$ has order at most 2 and hence it is contained in $Z(G)$, as required.

(2) Let $\langle a\rangle$ be a cyclic subgroup of $G$ of index less than 6 .

If $|G:\langle a\rangle|=2$ or $|G:\langle a\rangle|=3$, then the result follows from Proposition 2.4. Suppose, finally, that $|G:\langle a\rangle|=4$. If $\beta=0$ then the result follows by (1), and if $\langle a\rangle$ is maximal in $G$, then the result follows again from Proposition 2.4.

So suppose that $\beta>0$ and that $\langle a\rangle$ is not maximal in $G$. Then by Proposition $2.5 G$ has a normal cyclic Sylow 3 -subgroup $P$. Thus $G=P \rtimes D$, where $|D|=2^{\alpha}$. Obviously $P \leq\langle a\rangle$ and $D \simeq G / P$ has the cyclic subgroup $\langle a\rangle / P$ of index 4 . Hence by (1), $D^{\prime \prime} \leq Z(D)$. Now we have $G=P D, G^{\prime} \leq C_{G}(P)$ and $G^{\prime}=D^{\prime}[P, D]$, which implies that

$$
G^{\prime \prime}=D^{\prime \prime} \leq Z(D) \cap C_{G}(P) \leq Z(G)
$$

as required.

We also state the result of Ramanujan (see [8], page 46), which was mentioned in the introduction.

Proposition 2.7 (Ramanujan) If $q_{1}=2, q_{2}, \cdots, q_{n}, \cdots$ is the increasing sequence of all primes, then

$$
\prod_{i=1, \cdots, \infty} \frac{q_{i}^{2}+1}{q_{i}^{2}-1}=\frac{5}{2}
$$

This proposition implies the following lemma.

Lemma 2.8 Let $p_{2}, p_{3}, \ldots, p_{s}$ be primes satisfying $p_{2}<p_{3}<\cdots<p_{s}$. If $p_{2}>3$ then

$$
\prod_{i=2}^{s} \frac{p_{i}^{2}-1}{p_{i}^{2}+1}>\frac{5}{6}
$$


Proof. If $p_{2}>3$, then Proposition 2.7 implies that

$$
\frac{2^{2}+1}{2^{2}-1} \frac{3^{2}+1}{3^{2}-1} \prod_{i=2, \cdots, s} \frac{p_{i}^{2}+1}{p_{i}^{2}-1}<\frac{5}{2}
$$

Thus $\prod_{i=2, \cdots, s} \frac{p_{i}^{2}+1}{p_{i}^{2}-1}<\frac{6}{5}$, yielding

$$
\prod_{i=2, \cdots, s} \frac{p_{i}^{2}-1}{p_{i}^{2}+1}>\frac{5}{6}
$$

as required.

We shall also need some basic facts about $\psi\left(C_{n}\right)$.

\section{Lemma 2.9}

(1) If $P$ is a cyclic group of order $p^{r}$ for some prime $p$, then

$$
\psi(P)=\frac{p^{2 r+1}+1}{p+1}=\frac{p|P|^{2}+1}{p+1} .
$$

155

(2) Let $p_{1}<p_{2}<\cdots<p_{t}=p$ be the prime divisors of $n$ and denote the corresponding Sylow subgroups of $C_{n}$ by $P_{1}, P_{2}, \ldots, P_{t}$. Then

$$
\psi\left(C_{n}\right)=\prod_{i=1}^{t} \psi\left(P_{i}\right) \geq \frac{2}{p+1} n^{2}
$$

Proof.

(1) $\psi(P)=1+p \varphi(p)+p^{2} \varphi\left(p^{2}\right)+\cdots+p^{r} \varphi\left(p^{r}\right)=\frac{p^{2 r+1}+1}{p+1}=\frac{p|P|^{2}+1}{p+1}$.

(2) Since $C_{n}=P_{1} \times P_{2} \times \cdots \times P_{t}$, it follows by Lemma 2.2(3) that $\psi\left(C_{n}\right)=$ $\prod_{i=1}^{t} \psi\left(P_{i}\right)$. Since $p_{i+1} \geq p_{i}+1$ for all $i$ and $p_{1} \geq 2$, it follows by (1) that

$$
\psi\left(C_{n}\right)=\prod_{i=1}^{t} \frac{p_{i}\left|P_{i}\right|^{2}+1}{p_{i}+1}>\prod_{i=1}^{t} \frac{p_{i}}{p_{i}+1}\left|P_{i}\right|^{2} \geq \frac{2}{p+1} n^{2} .
$$


Finally, we state Corollary B from [1].

Proposition 2.10 If $P$ is a cyclic normal Sylow p-subgroup of a finite group $G$, then

$$
\psi(G) \leq \psi(P) \psi(G / P),
$$

with equality if and only if $P$ is central in $G$.

\section{Proofs of the main results.}

Since we are using the result of Theorem 3 for the proof of Theorem 1, we Theorem 1.

Proof of Theorem 3. We need to prove that if $\psi(G) \geq \frac{1}{q-1} \psi\left(C_{n}\right)$, then $G \cong C_{n}$.

Clearly $\psi\left(C_{n}\right)>n \varphi(n)$ and by Lemma $2.1 \varphi(n) \geq(q-1) n / p$, where $p$ denotes the largest prime divisor of $n$. Hence by our assumptions $\psi(G)>$ $\frac{n(q-1) n}{(q-1) p}=n^{2} / p$, which implies that there exists $x \in G$ with $o(x)>n / p$. Thus $[G:\langle x\rangle]\left\langle p\right.$ and $\langle x\rangle$ contains a Sylow $p$-subgroup $P$ of $G$. Since $\langle x\rangle \leq N_{G}(P)$, it follows that $P$ is a cyclic normal subgroup of $G$ and Proposition 2.10 implies that

$$
\psi(P) \psi(G / P) \geq \psi(G) \geq \frac{1}{q-1} \psi\left(C_{p^{r}}\right) \psi\left(C_{n / p^{r}}\right),
$$

where $p^{r}=|P|$. Since $P \cong C_{p^{r}}$, cancellation yields

$$
\psi(G / P) \geq \frac{1}{q-1} \psi\left(C_{n / p^{r}}\right)
$$

If $n=p^{r}, p$ a prime, then the existence of $x \in G$ satisfying $o(x)>n / p$ implies that $o(x)=n$ and $G$ is cyclic, as required. So we may assume that $n$ is divisible by exactly $k$ different primes with $k>1$. Applying induction with respect to $k$, we may assume that the theorem holds for groups of order which has less than $k$ distinct prime divisors. Since $|G / P|$ has $k-1$ distinct prime divisors and $G / P$ satisfies our assumptions, it follows that $G / P$ is cyclic and 
$G=P \rtimes F$, with $F \cong G / P$ and $F \neq 1$. Notice that $n=|P||F|, P$ and $F$ are both cyclic and $(|P|,|F|)=1$. Hence $\psi\left(C_{n}\right)=\psi(P) \psi(F)$.

If $C_{F}(P)=F$, then $G=P \times F$ and $G$ is cyclic, as required.

So it suffices to prove that if $C_{F}(P)=Z<F$, then $\psi(G)<(1 /(q-1)) \psi\left(C_{n}\right)$, contrary to our assumptions. It follows by Lemma 2.2(5) that

$$
\psi(G)=\psi(P) \psi(Z)+|P| \psi(F \backslash Z)<\psi(P) \psi(Z)+|P| \psi(F)
$$

Hence

$$
\psi(G)<\psi(P) \psi(F)\left(\frac{\psi(Z)}{\psi(F)}+\frac{|P|}{\psi(P)}\right)=\psi\left(C_{n}\right)\left(\frac{\psi(Z)}{\psi(F)}+\frac{|P|}{\psi(P)}\right) .
$$

Notice first that since $P$ is a cyclic $p$-group, we have

$$
\frac{|P|}{\psi(P)}=\frac{|P|(p+1)}{p|P|^{2}+1}<\frac{p+1}{p|P|} \leq \frac{p+1}{p^{2}}<\frac{p+1}{p^{2}-1}=\frac{1}{p-1} \leq \frac{1}{q} .
$$

Next notice that $Z$ is a proper subgroup of the cyclic group $F$ and $\psi(F)$ is a product of $\psi(S)$, with $S$ running over the Sylow subgroups of $F$. Since also $\psi(Z)$ is a similar product, and since at least one Sylow subgroup of $Z$, say Sylow $r$-subgroup $R_{Z}$, is properly contained in the Sylow $r$-subgroup $R_{F}$ of $F$ of order $r^{s}$, it follows that

$$
\frac{\psi(Z)}{\psi(F)} \leq \frac{\psi\left(R_{Z}\right)}{\psi\left(R_{F}\right)} \leq \frac{r^{2(s-1)+1}+1}{r^{2 s+1}+1}
$$

Since $r \geq q$ and $s \geq 1$, we get

$$
\frac{\psi(Z)}{\psi(F)} \leq \frac{r^{2 s-1}+1}{r^{2 s+1}+1}<\frac{1}{q(q-1)}
$$

Therefore

$$
\psi(G)<\psi\left(C_{n}\right)\left(\frac{\psi(Z)}{\psi(F)}+\frac{|P|}{\psi(P)}\right)<\psi\left(C_{n}\right)\left(\frac{1}{q(q-1)}+\frac{1}{q}\right)=\psi\left(C_{n}\right) \frac{1}{q-1}
$$

a contradiction.

The proof is now complete.

We continue with the proof of Theorem 1 . 
Proof of Theorem 1. Throughout this proof $G$ denotes a non-cyclic finite group of order $n$ satisfying

$$
\psi(G)>\frac{7}{11} \psi\left(C_{n}\right)
$$

Let $p_{1}<p_{2}<\cdots<p_{t}=p$ be the prime divisors of $n$ and denote the corresponding Sylow subgroups of $C_{n}$ by $P_{1}, P_{2}, \ldots, P_{t}$. By Lemma $2.9 \psi\left(C_{n}\right) \geq \frac{2}{p+1} n^{2}$, so our assumptions imply that $G$ satisfies

$$
\psi(G)>\frac{7}{11} \psi\left(C_{n}\right) \geq \frac{14}{11(p+1)} n^{2} .
$$

Our aim is to reach a contradiction. Our proof is by induction on the size of $p$.

By our assumptions there exists $x \in G$ with $o(x)>\frac{14}{11(p+1)} n$, which implies that

$$
[G:\langle x\rangle]<\frac{11(p+1)}{14} .
$$

Suppose, first, that $p=2$. Then $G$ is a 2-group and $[G:\langle x\rangle]<\frac{33}{14}$. Thus $[G:\langle x\rangle]=2, n \geq 4$ and $n^{2} \geq 16$, implying that

$\psi(G) \leq \psi\left(C_{n / 2}\right)+\left(\frac{n}{2}\right)^{2}=\frac{2(n / 2)^{2}+1}{3}+\frac{n^{2}}{4}=\frac{5}{12} n^{2}+\frac{1}{3} \leq\left(\frac{7}{11}\right)\left(\frac{2 n^{2}+1}{3}\right)=\left(\frac{7}{11}\right) \psi\left(C_{n}\right)$,

a contradiction.

Next assume that $p=3$ and $[G:\langle x\rangle]<\frac{44}{14}$. If $G$ is a 3-group, then Theorem 3 yields $\psi(G)<\frac{1}{2} \psi\left(C_{n}\right)<\frac{7}{11} \psi\left(C_{n}\right)$, a contradiction. So we may assume that $n=2^{a} 3^{b}$ for some positive integers $a$ and $b$. Since $[G:\langle x\rangle]<\frac{44}{14}$, it follows that $[G:\langle x\rangle] \leq 3$. Hence either $[G:\langle x\rangle]=2$ or $[G:\langle x\rangle]=3$. Notice for later reference that if $n=2^{a} 3^{b}$, then

$$
\begin{gathered}
\frac{7}{11} \psi\left(C_{n}\right) \\
=\frac{7}{11} \psi\left(C_{2^{a}}\right) \psi\left(C_{3^{b}}\right)=\frac{7}{11}\left(\frac{2^{2 a+1}+1}{3}\right)\left(\frac{3^{2 b+1}+1}{4}\right) \\
=\frac{7}{22} 2^{2 a} 3^{2 b}+\frac{7}{66} 2^{2 a}+\frac{7}{44} 3^{2 b}+\frac{7}{132} .
\end{gathered}
$$

Suppose first that $[G:\langle x\rangle]=2$. Then $\langle x\rangle$ contains a cyclic Sylow 3-subgroup $P$ of $G$ and since $\langle x\rangle \leq C_{G}(P)$, it follows that $P$ is normal in $G$. 
If there exists $y \in G \backslash\langle x\rangle$ with $[G:\langle y\rangle]=2$, then $y \in C_{G}(P)$ and hence $P \leq Z(G)$. Thus $G=P \times Q$, where $Q$ is a non-cyclic Sylow 2-subgroup of $G$ and it follows by our result for $p=2$ that

$$
\psi(G)=\psi(P) \psi(Q) \leq \psi(P)\left(\frac{7}{11}\right) \psi\left(C_{|Q|}\right)=\left(\frac{7}{11}\right) \psi\left(C_{n}\right),
$$

a contradiction.

So suppose that $o(y) \leq \frac{n}{3}$ for all $y \in G \backslash\langle x\rangle$. Since $a \geq 1$ and $b \geq 1$, we obtain the following final contradiction with respect to $[G:\langle x\rangle]=2$ :

$$
\begin{gathered}
\psi(G) \leq \psi\left(C_{\frac{n}{2}}\right)+\left(\frac{n}{2}\right)\left(\frac{n}{3}\right)=\psi\left(C_{2^{a-1}}\right) \psi\left(C_{3^{b}}\right)+\frac{n^{2}}{6}=\left(\frac{2^{2 a-1}+1}{3}\right)\left(\frac{3^{2 b+1}+1}{4}\right)+\frac{n^{2}}{6} \\
=\left(\frac{1}{6}\right)\left(\frac{3}{4}\right) 2^{2 a} 3^{2 b}+\frac{2^{2 a}}{24}+\frac{3^{2 b}}{4}+\frac{1}{12}+\frac{2^{2 a} 3^{2 b}}{6}=\frac{7}{24} 2^{2 a} 3^{2 b}+\frac{2^{2 a}}{24}+\frac{3^{2 b}}{4}+\frac{1}{12} \\
=\frac{7}{22} 2^{2 a} 3^{2 b}+\frac{7}{66} 2^{2 a}+\frac{7}{44} 3^{2 b}+\frac{7}{132} \\
+\left(\frac{7}{24}-\frac{7}{22}\right) 2^{2 a} 3^{2 b}+\left(\frac{1}{24}-\frac{7}{66}\right) 2^{2 a}+\left(\frac{1}{4}-\frac{7}{44}\right) 3^{2 b}+\left(\frac{11}{132}-\frac{7}{132}\right) \\
<\frac{7}{11} \psi\left(C_{n}\right)-\frac{7}{264} 2^{2 a} 3^{2 b}+\frac{1}{11} 3^{2 b}+\frac{4}{132} \\
\leq \frac{7}{11} \psi\left(C_{n}\right)-\frac{7}{66} 3^{2 b}+\frac{6}{66} 3^{2 b}+\frac{2}{66}<\frac{7}{11} \psi\left(C_{n}\right) .
\end{gathered}
$$

We approach now the second possibility: $p=3$ and $[G:\langle x\rangle]=3$. By the previous arguments we may assume that no element of $G$ is of order $\frac{n}{2}$ and hence $o(y) \leq \frac{n}{3}$ for all $y \in G$. By considering elements of $G$ belonging to $\langle x\rangle$ and those outside it, and recalling that $b \geq 1$, we obtain the following contradiction:

$$
\begin{gathered}
\psi(G) \leq \psi\left(C_{2^{a}}\right) \psi\left(C_{3^{b-1}}\right)+2\left(\frac{n}{3}\right)^{2}=\left(\frac{2^{2 a+1}+1}{3}\right)\left(\frac{3^{2 b-1}+1}{4}\right)+\frac{2}{9} n^{2} \\
=\frac{1}{18} 2^{2 a} 3^{2 b}+\frac{1}{6} 2^{2 a}+\frac{1}{36} 3^{2 b}+\frac{1}{12}+\frac{2}{9} 2^{2 a} 3^{2 b}=\frac{5}{18} 2^{2 a} 3^{2 b}+\frac{1}{6} 2^{2 a}+\frac{1}{36} 3^{2 b}+\frac{1}{12} \\
=\frac{7}{22} 2^{2 a} 3^{2 b}+\frac{7}{66} 2^{2 a}+\frac{7}{44} 3^{2 b}+\frac{7}{132} \\
+\left(\frac{5}{18}-\frac{7}{22}\right) 2^{2 a} 3^{2 b}+\left(\frac{1}{6}-\frac{7}{66}\right) 2^{2 a}+\left(\frac{1}{36}-\frac{7}{44}\right) 3^{2 b}+\left(\frac{11}{132}-\frac{7}{132}\right) \\
<\frac{7}{11} \psi\left(C_{n}\right)-\frac{4}{99} 2^{2 a} 3^{2 b}+\frac{2}{33} 2^{2 a}+\frac{1}{33} \\
\leq \frac{7}{11} \psi\left(C_{n}\right)-\frac{36}{99} 2^{2 a}+\frac{6}{99} 2^{2 a}+\frac{1}{33}<\frac{7}{11} \psi\left(C_{n}\right) .
\end{gathered}
$$


So assume, finally, that $p>3$ and that the theorem holds for smaller values of $p$. Then

$$
[G:\langle x\rangle]<\frac{11(p+1)}{14} \leq p
$$

and $\langle x\rangle$ contains a cyclic Sylow $p$-subgroup $P$ of $G$. Since $\langle x\rangle \leq N_{G}(P)$, it follows that $P$ is a cyclic normal subgroup of $G$ and Proposition 2.10 implies that

$$
\psi(P) \psi(G / P) \geq \psi(G)>\frac{7}{11} \psi\left(C_{p^{r}}\right) \psi\left(C_{n / p^{r}}\right),
$$

where $p^{r}=|P|$. Since $P \cong C_{p^{r}}$, cancellation yields

$$
\psi(G / P)>\frac{7}{11} \psi\left(C_{n / p^{r}}\right) .
$$

Since the maximal prime dividing $n / p^{r}$ is smaller than $p$, our induction hypothesis implies that $G / P$ is cyclic and $G=P \rtimes F$, with $F \cong G / P$ and $F \neq 1$. Notice that $n=|P||F|$, with both $P$ and $F$ being cyclic, and $(|P|,|F|)=1$. Hence $\psi\left(C_{n}\right)=\psi(P) \psi(F)$.

If $C_{F}(P)=F$, then $G=P \times F$ and $G$ is a cyclic, a contradiction. So suppose that $C_{F}(P)=Z<F$. Lemma 2.2(5) then implies that

$$
\psi(G)<\psi(P) \psi(F)\left(\frac{\psi(Z)}{\psi(F)}+\frac{|P|}{\psi(P)}\right)=\psi\left(C_{n}\right)\left(\frac{\psi(Z)}{\psi(F)}+\frac{|P|}{\psi(P)}\right) .
$$

Notice first that since $P$ is a cyclic $p$-group and $p>3$, we have

$$
\frac{|P|}{\psi(P)}=\frac{|P|(p+1)}{p|P|^{2}+1}<\frac{p+1}{p|P|} \leq \frac{p+1}{p^{2}} \leq \frac{6}{25}<\frac{1}{4} .
$$

Next notice that $Z$ is a proper subgroup of the cyclic group $F$ and $\psi(F)$ is a product of $\psi(S)$, with $S$ running over the Sylow subgroups of $F$. Since also $\psi(Z)$ is a similar product, and since at least one Sylow subgroup of $Z$, say Sylow $r$-subgroup $R_{Z}$, is properly contained in the Sylow $r$-subgroup $R_{F}$ of $F$ of order $r^{s}$, it follows that

$$
\frac{\psi(Z)}{\psi(F)} \leq \frac{r^{2(s-1)+1}+1}{r^{2 s+1}+1}
$$

But $r \geq 2$ and $s \geq 1$, so

$$
\frac{r^{2(s-1)+1}+1}{r^{2 s+1}+1} \leq \frac{1}{r+1}
$$


since this inequality is equivalent to $1 \leq r^{2 s-2}\left(r^{2}-r-1\right)$, which is true. Hence

$$
\frac{\psi(Z)}{\psi(F)} \leq \frac{1}{r+1} \leq \frac{1}{3}
$$

and

$$
\psi(G)<\psi\left(C_{n}\right)\left(\frac{\psi(Z)}{\psi(F)}+\frac{|P|}{\psi(P)}\right)<\psi\left(C_{n}\right)\left(\frac{1}{3}+\frac{1}{4}\right)=\psi\left(C_{n}\right) \frac{7}{12}<\psi\left(C_{n}\right) \frac{7}{11},
$$

a final contradiction.

The proof is now complete.

Next we prove Proposition 2.

Proof of Proposition 2. We start with the proof of the first equality:

$$
\psi\left(C_{n}\right)=\psi\left(C_{4 k}\right)=\psi\left(C_{4}\right) \psi\left(C_{k}\right)=\frac{32+1}{2+1} \psi\left(C_{k}\right)=11 \psi\left(C_{k}\right) .
$$

Next we prove the second equality:

$$
\psi\left(C_{2 k} \times C_{2}\right)=\psi\left(C_{k} \times C_{2} \times C_{2}\right)=\psi\left(C_{k}\right) \psi\left(C_{2} \times C_{2}\right)=7 \psi\left(C_{k}\right) .
$$

The claim follows.

We continue with a proof of Theorem 6 .

Proof of Theorem 6. Since $\psi\left(C_{n}\right)>n \varphi(n)$ and by Lemma $2.1 \varphi(n) \geq \frac{(q-1) n}{p}$, it follows by our assumptions that $\psi(G)>\frac{n^{2}}{2 p}$. Hence there exists $x \in G$ such that $o(x)>\frac{n}{2 p}$ and

$$
[G:\langle x\rangle]<2 p .
$$

First we shall prove that $G$ is solvable. Let $k$ be the number of prime divisors of $n$. Our proof is by induction on $k$.

If $k=1$, then $G$ is a $p$-group, hence solvable, as claimed. So assume that $k>1$ and that the claim holds for $k-1$.

If $p \mid[G:\langle x\rangle]$, then $[G:\langle x\rangle]=p$ and $\langle x\rangle$ is a cyclic maximal subgroup of $G$. Hence by Proposition $2.3 G$ is solvable, as claimed. 
So suppose that $p \nmid[G:\langle x\rangle]$. Then $\langle x\rangle$ contains a cyclic Sylow $p$-subgroup $P$ of $G$.

If $P$ is normal in $G$, then Proposition 2.10 and our assumptions imply that

$$
\psi(P) \psi(G / P) \geq \psi(G) \geq \frac{1}{2(q-1)} \psi\left(C_{|P|}\right) \psi\left(C_{|G / P|}\right),
$$

and since $\psi(P)=\psi\left(C_{|P|}\right)$, it follows that

$$
\psi(G / P) \geq \frac{1}{2(q-1)} \psi\left(C_{|G / P|}\right) .
$$

Hence, by induction, $G / P$ is solvable and so $G$ is solvable, as claimed.

Suppose, finally, that $P$ is not normal in $G$. Since $\langle x\rangle$ is a subgroup of $N_{G}(P)$, it follows that $\left[G: N_{G}(P)\right]<2 p$ and hence

$$
\left[G: N_{G}(P)\right]=p+1
$$

Since

$$
\left[N_{G}(P):\langle x\rangle\right]=\frac{[G:\langle x\rangle]}{\left[G: N_{G}(P)\right]} \leq \frac{2 p}{p+1}<2,
$$

it follows that $N_{G}(P)=\langle x\rangle$ is a cyclic maximal subgroup of $G$. Hence $G$ is solvable by Proposition 2.3 and the proof of our claim is complete.

We proceed with the proof of the theorem. As shown above, there exists $x \in G$ such that $[G:\langle x\rangle]<2 p$. By Proposition 2.5 this implies that either $G$ has a normal cyclic Sylow $p$-subgroup or $\langle x\rangle$ is a maximal subgroup of $G$ of index either $p$ or $p+1$. In either case, the Sylow $p$-subgroups of $G$ contain a cyclic subgroup of index $p$, as required.

If $G$ has a normal cyclic Sylow $p$-subgroup, then (1) holds. If $\langle x\rangle$ is a maximal subgroup of $G$ of index $p$, then it contains a Sylow $q$-subgroup $Q$ of $G$. Since $Q$ is cyclic and $q$ is the smallest prime divisor of $n$, it follows by Theorem 10.1.9 in [5] that $G$ is $q$-nilpotent. Moreover, by Proposition $2.4 G^{\prime \prime} \leq Z(G)$ and (2) holds.

Finally, if $\langle x\rangle$ is a maximal subgroup of $G$ of index $p+1$, then it contains a cyclic Sylow $p$-subgroup $P$ of $G$. If $P$ is normal, then (1) holds. So suppose that

$P$ is not normal in $G$. Since $\langle x\rangle \leq N_{G}(P)$, it follows that $\left[G: N_{G}(P)\right]<2 p$, 
which implies that $\left[G: N_{G}(P)\right]=p+1$. As shown above $N_{G}(P)=\langle x\rangle$ and hence $N_{G}(P)=C_{G}(P)$. Thus $G$ is $p$-nilpotent by Burnside's theorem, and since $\langle x\rangle$ is a cyclic maximal subgroup of $G$, Proposition 2.4 implies that $G^{\prime \prime} \leq Z(G)$ and (3) holds. The proof of the theorem is complete.

We continue with a proof of a sufficient condition for the solvability of a finite group $G$.

Proof of Theorem 10. Suppose that

$$
\psi(G) \geq \frac{3}{5} n \varphi(n)
$$

and let $p_{1}$ be the maximal prime dividing $n$. By Lemma $2.1 \varphi(n) \geq n / p_{1}$, so by our assumptions $\psi(G) \geq \frac{3}{5} n^{2} / p_{1}$. Hence there exists an element $x$ of $G$ with $o(x)>\frac{3}{5} n / p_{1}$ and

$$
|G:\langle x\rangle|<\frac{5}{3} p_{1}<2 p_{1} .
$$

It follows by Proposition 2.5 that either $G$ is solvable, or $G$ has a normal cyclic Sylow $p_{1}$-subgroup $P_{1}$.

We prove first that $G$ is solvable. Clearly we may assume that $n$ is divisible by at least three different primes and hence $p_{1} \geq 5$. We may also assume that $G$ has a normal cyclic Sylow $p_{1}$-subgroup $P_{1}$. Hence $G=P_{1} \rtimes H$ for a suitable subgroup $H$ of $G$, and by Proposition $2.10 \psi(G) \leq \psi\left(P_{1}\right) \psi(H)$. Thus

$$
\psi(H) \geq \frac{\psi(G)}{\psi\left(P_{1}\right)} .
$$

Let $|H|=h$. Then $n=h\left|P_{1}\right|$ and $\varphi(n)=\varphi(h) \varphi\left(\left|P_{1}\right|\right)=\varphi(h)\left(p_{1}-1\right)\left|P_{1}\right| / p_{1}$. Recalling that $p_{1} \geq 5$, we get

$$
\begin{gathered}
\psi(H) \geq\left(\frac{3}{5}\right)\left(\frac{n \varphi(n)\left(p_{1}+1\right)}{\left(p_{1}\left|P_{1}\right|^{2}+1\right)}\right)=\left(\frac{3}{5}\right)\left(\frac{h \varphi(h)\left|P_{1}\right|\left(p_{1}-1\right)\left|P_{1}\right|\left(p_{1}+1\right)}{p_{1}\left(p_{1}\left|P_{1}\right|^{2}+1\right)}\right) \\
>\left(\frac{3}{5}\right)\left(\frac{h \varphi(h)\left|P_{1}\right|^{2}\left(p_{1}^{2}-1\right)}{\left(p_{1}^{2}+1\right)\left|P_{1}\right|^{2}}\right)=\left(\frac{3}{5}\right)\left(\frac{h \varphi(h)\left(p_{1}^{2}-1\right)}{\left(p_{1}^{2}+1\right)}\right) \geq h \varphi(h)\left(\frac{3}{5}\right)\left(\frac{24}{26}\right)>h \varphi(h) \frac{1}{2} .
\end{gathered}
$$

Let $p_{2}$ be the maximal prime dividing $h$. Then by Lemma $2.1 \psi(H)>\frac{1}{2} \frac{h^{2}}{p_{2}}$, and hence there exists an element $y \in H$ satisfying $o(y)>\frac{1}{2} \frac{h}{p_{2}}$. Thus $|H:\langle y\rangle|<2 p_{2}$ 
and by Proposition 2.5 either $H$ is solvable or there exists a normal cyclic Sylow $p_{2}$-subgroup $P_{2}$ of $H$. If $H$ is solvable, then also $G$ is solvable, as required.

So suppose that there exists a normal cyclic Sylow $p_{2}$-subgroup $P_{2}$ of $H$. Then $G=P_{1} \rtimes\left(P_{2} \rtimes V\right)$ for a suitable subgroup $V$ of $G$.

Now, let $p_{1}>p_{2}>\cdots>p_{t}>3$ be primes and suppose that

$$
G=P_{1} \rtimes\left(P_{2} \rtimes\left(\cdots \rtimes\left(P_{t} \rtimes K\right)\right)\right),
$$

where $P_{i}$ are cyclic Sylow $p_{i}$-subgroups of $G$ and $K$ is a suitable subgroup of $G$. Write $|K|=k$ and suppose that $t$ is maximal under these conditions. It follows from Proposition 2.10 that

$$
\psi(G) \leq \psi\left(P_{1}\right) \psi\left(P_{2}\right) \cdots \psi\left(P_{t}\right) \psi(K),
$$

and hence, noting that $p_{t}>3$ and using Lemma 2.9 , we get

$$
\begin{gathered}
\psi(K) \geq \frac{\psi(G)}{\psi\left(P_{1}\right) \psi\left(P_{2}\right) \cdots \psi\left(P_{t}\right)} \geq \frac{3}{5} n \varphi(n) \prod_{i=1}^{t} \frac{\left(p_{i}+1\right)}{\left(p_{i}\left|P_{i}\right|^{2}+1\right)} \\
=\frac{3}{5} k \varphi(k) \prod_{i=1}^{t} \frac{\left|P_{i}\right|\left(p_{i}-1\right)\left|P_{i}\right|\left(p_{i}+1\right)}{p_{i}\left(p_{i}\left|P_{i}\right|^{2}+1\right)}=\frac{3}{5} k \varphi(k) \prod_{i=1}^{t} \frac{\left|P_{i}\right|^{2}\left(p_{i}^{2}-1\right)}{p_{i}\left(p_{i}\left|P_{i}\right|^{2}+1\right)} \\
>\frac{3}{5} k \varphi(k) \prod_{i=1}^{t} \frac{p_{i}^{2}-1}{p_{i}^{2}+1}>k \varphi(k)\left(\frac{3}{5}\right)\left(\frac{5}{6}\right)=k \varphi(k) \frac{1}{2} .
\end{gathered}
$$

Let $p_{t+1}$ be the maximal prime dividing $k$. Then by Lemma $2.1 \psi(K)>$ $\frac{1}{2} \frac{k^{2}}{p_{t+1}}$, and there exists an element $v \in K$ satisfying $o(v)>\frac{1}{2} \frac{k}{p_{t+1}}$. Thus $\mid K$ : $\langle v\rangle \mid<2 p_{t+1}$ and by Proposition 2.5 either $K$ is solvable, or there exists a normal cyclic Sylow $p_{t+1}$-subgroup $P_{t+1}$ of $K$. In the former case, $K$ is solvable, and hence also $G$ is solvable, as required. If, on the other hand, the latter case occurs, then $K=P_{t+1} \rtimes W$ for a suitable subgroup $W$ of $K$, and by the maximality of $t, p_{t+1} \leq 3$. Thus $K$ is a $(2,3)$-group, hence solvable, so also $G$ is solvable. The proof of the solvability of $G$ is now complete.

Moreover, we have proved that

$$
G=P_{1} \rtimes\left(P_{2} \rtimes\left(\cdots\left(P_{t} \rtimes K\right)\right)\right),
$$


where $P_{i}$ are cyclic Sylow $p_{i}$-subgroups of $G$, and either $K$ has a cyclic maximal subgroup or $|K|=2^{\alpha} 3^{\beta}$ and $K$ has a cyclic subgroup of index $<6$. We shall show now by induction on $t$ that these assumptions imply that $G^{\prime \prime} \leq Z(G)$. If $t=0$, then the result follows from Proposition 2.4 and Proposition 2.6(2). So suppose that $t>0$ and set $H=\left(P_{2} \rtimes \cdots\left(P_{t} \rtimes K\right)\right)$. It follows by induction that $H^{\prime \prime} \leq Z(H)$. Since $G=P_{1} \rtimes H$, where $P_{1}$ is cyclic group, we have $G^{\prime} \leq C_{G}\left(P_{1}\right)$ and $G^{\prime}=H^{\prime}\left[P_{1}, H\right]$. Hence

$$
G^{\prime \prime}=H^{\prime \prime} \leq Z(H) \cap C_{G}\left(P_{1}\right) \leq Z(G),
$$

which completes the proof of Theorem 10 .

Our last proof is that of Theorem 11, concerning groups of order $n$ satisfying ${ }_{245} \psi(G) \geq \frac{1}{q} n \varphi(n)$.

Proof of Theorem 11. Suppose that $G$ is a group of order $n$ and it satisfies $\psi(G) \geq \frac{1}{q} n \varphi(n)$. Since by Lemma $2.1 \varphi(n) \geq \frac{(q-1) n}{p}$, it follows by our assumptions that $\psi(G) \geq \frac{(q-1) n^{2}}{q p}$. Thus there exists $x \in G$ with $o(x)>\frac{(q-1) n}{q p}$ and

$$
[G:\langle x\rangle]<\frac{q}{q-1} p \leq 2 p .
$$

Hence by Proposition 2.5 either $G$ has a normal cyclic Sylow $p$-subgroup, or it is a solvable group with a cyclic maximal subgroup of index either $p$ or $p+1$, as required. The proof of the theorem is now complete.

\section{References}

\section{References}

[1] H. Amiri, S.M. Jafarian Amiri, I.M. Isaacs, Sums of element orders in finite groups, Comm. Algebra 37, (2009), 2978-2980.

[2] H. Amiri, S.M. Jafarian Amiri, Sum of element orders on finite groups of the same order, J. Algebra Appl. 10 (2), (2011), 187-190. 
[3] M. Garonzi, M. Patassini, Inequalities detecting structural properties of a finite group arXiv:1503.00355v2, (2015).

[4] S.M. Jafarian Amiri, Second maximum sum of element orders on finite nilpotent groups, Comm. Algebra 41 (6), (2013), 2055-2059.

[5] S.M. Jafarian Amiri, M. Amiri, Second maximum sum of element orders on finite groups, J. Pure Appl. Algebra 218 (3), (2014), 531-539.

[6] D. Gorenstein, Finite groups, Harper and Row, New York, 1968.

[7] I.N. Herstein, A remark on finite groups, Proc. Amer. Math. Soc. 9, (1958), $255-257$.

[8] F. Le Lionnais, Les nombres remarquables, Hermann, Paris, 1983.

[9] 9 D.V. Lytkina, V.D. Mazurov, Groups with Given Element Orders, J. Siberian Federal University Math. 7 (2), (2014), 191-203.

[10] D.J.S. Robinson, A course in the theory of groups, 2nd edn., Springer, New York, 1996.

[11] R. Shen, G. Chen, C. Wu, On groups with the Second Largest Value of the Sum of Element Orders, Comm. Algebra 43 (6), (2015), 2618-2631. 